\title{
Synergy study on charge transport dynamics in hybrid organic solar cell: photocurrent mapping and performance analysis under local spectrum
}

\begin{abstract}
Charge transport dynamics in $\mathrm{ZnO}$ based inverted organic solar cell (IOSC) has been characterized with transient photocurrent spectroscopy and localised photocurrent mappingatomic force microscopy. The value of maximum exciton generation rate was found to vary from $2.6 \times 1027 \mathrm{~m}-3 \mathrm{~s}-1$ (Jsat $=79.7 \mathrm{~A} \mathrm{~m}-2)$ to $2.9 \times 1027 \mathrm{~m}-3 \mathrm{~s}-1 \quad(\mathrm{Jsat}=90.8 \mathrm{~A} \mathrm{~m}-2)$ for devices with power conversion efficiency ranging from 2.03 to $2.51 \%$. These results suggest that nanorods served as an excellent electron transporting layer that provides efficient charge transport and enhances IOSC device performance. The photovoltaic performance of OSCs with various growth times of $\mathrm{ZnO}$ nanorods have been analysed for a comparison between AM1.5G spectrum and local solar spectrum. The simulated PCE of all devices operating under local spectrum exhibited extensive improvement with the gain of $13.3-13.7 \%$ in which the $\mathrm{ZnO}$ nanorods grown at 15 min possess the highest PCE under local solar with the value of $2.82 \%$.
\end{abstract}

Keyword: ZnO; Hydrothermal; Defect; Nanorod; Inverted; Energy band gap 